\title{
Role of Proton Beam Therapy in Current Day Radiation Oncology Practice
}

\author{
${ }^{1}$ Department of Radiation Oncology, MAX Super Speciality Hospital \\ Patparganj and Vaishali, New Delhi, India \\ ${ }^{2}$ Department of Radiation Oncology, Dharamshila Narayana \\ Superspeciality Hospital, New Delhi, India \\ ${ }^{3}$ Department of Radiation Oncology, Vardhaman Mahavir Medical \\ College and Safdarjung Hospital, New Delhi, India \\ Asian J Oncol:2020;6:97-106
}

Gagan Saini ${ }^{1}$ Rashmi Shukla ${ }^{1} \quad$ Kanika S. Sood ${ }^{2}$ Sujit K. Shukla ${ }^{3}$ Ritu Chandra

\author{
Address for correspondence Rashmi Shukla, MBBS, DNB, \\ Department of Radiation Oncology, MAX Super Speciality Hospital, \\ New Delhi 110092, India (e-mail: drrash11@gmail.com).
}

\begin{abstract}
Keywords

- Braggs peak

- pediatric malignancies

- normal tissue sparing

- PBT

Proton beam therapy (PBT), because of its unique physics of no-exit dose deposition in the tissue, is an exciting prospect. The phenomenon of Bragg peak allows protons to deposit their almost entire energy towards the end of the path of the proton and stops any further dose delivery. Braggs peak equips PBT with superior dosimetric advantage over photons or electrons because PBT doesn't traverse the target/body but is stopped sharply at an energy dependent depth in the target/body. It also has no exit dose. Because of no exit dose and normal tissue sparing, PBT is hailed for its potential to bring superior outcomes. Pediatric malignancies is the most common malignancy where PBT have found utmost application. Nowadays, PBT is also being used in the treatment of other malignancies such as carcinoma prostate, carcinoma breast, head and neck malignancies, and gastrointestinal (GI) malignancies. Despite advantages of PBT, there is not only a high cost of setting up of PBT centers but also a lack of definitive phase-III data. Therefore, we review the role of PBT in current day practice of oncology to bring out the nuances that must guide the practice to choose suitable patients for PBT.
\end{abstract}

\section{Introduction}

It is said that no other branch of medicine has benefitted more from technological innovations in the last two decades as the branch of oncology. The same is reflected in intensified research in radiation oncology in the last two decades. ${ }^{1}$ It is estimated that up to 60 to $70 \%$ patients requiring cancer treatments need radiation therapy. ${ }^{2}$ Over a century, technical advancements in radiation oncology, like three-dimensional conformal radiotherapy (3D-CRT), intensity modulated radiotherapy (IMRT), volumetric modulated radiotherapy (VMAT), and image-guided radiotherapy (IGRT) have facilitated the delivery of photon therapy with precision. However, the dose deposited in the surrounding normal tissues albeit less still remains a concern in photon therapy. ${ }^{3}$ Proton beam therapy (PBT), because of its unique physics of no-exit dose deposition in the tissues, is an exciting prospect., ${ }^{4,5}$ PBT was first proposed by Wilson ${ }^{6}$ in 1946. Later, the Lawrence-Berkeley National Laboratory published their first PBT patient series. ${ }^{7}$ Thus far, PBT has used in the clinical setting for over 60 years, and has been applied to $10 \mathrm{~s}$ of thousands of patients with different types of cancers. ${ }^{8}$

The clinical benefits of PBT include a potential for fewer side effects compared with photon therapy. However, the exact role of PBT remains controversial. This is primarily due to a nonexistence of clear cut benefit for every clinical situation and the high treatment costs associated with the cost of proton facility building and maintenance. More research, studies and discussions are required to address the use of PBT in several types of cancer because of the potential of maintaining a relatively higher quality of life of patients while achieving cure. The aim of this review was to report the characteristics and current developments in PBT.
DOI https://doi.org/

$10.1055 / \mathrm{s}-0040-1713703$ ISSN 2454-6798.
License terms

(1) (1) $\Theta \circledast$ 


\section{Biology of Proton Beam Therapy}

All through the history of radiation therapy, patients have been treated with photon beams which are gamma rays or $\mathrm{X}$-rays. Both gamma rays and X-rays are physically one and the same thing and differ only in their method of generation, where gamma rays are generated from naturally radioactive elements like Cobalt-60 and the X-rays are produced via man-made linear accelerators. The growth of radiation oncology has been on various ways of administering these photon beams. These photons have been used with basic shielding of critical areas in early years or later by beam shaping with 3D-CRT. Now, these photons are used by modulating their deposition on the target by firing them in a controlled manner from all directions with a sophisticated computerized controlled planning and execution, this is the principle behind IMRT and VMAT. These photons have been used for treatment not just by employing better shielding and modulation but also by altering the dose and fractionation which can give a beneficial biological effect, for example, hypofractionation, hyperfractionation, integrated boost techniques, and stereotactic body radiotherapy (SBRT).

Now with PBT, we have a different beam altogether from the traditional photon beam. It is for this reason that it behaves very differently from photons. While the traditional photons are almost weightless, the proton is almost 800 times heavier than electron. This large mass gives a proton its momentum that gets quickly dissipated after traveling a defined distance in patient's body, this stops the proton from travelling a large distance in the patient's body. This leads to a sharp rise in energy deposition toward the end of the path of the proton and stops any further dose delivery. This phenomenon is referred to as the Bragg peak. ${ }^{9}$ Bragg's peak equips PBT with superior dosimetric advantage over photons or electrons because PBT doesn't traverse the target/body but is stopped sharply at an energy-dependent depth in the target/body. It also has no exit dose ( - Fig. 1). Conversely, when treated with photons the normal tissues do receive low radiation doses and the benefit of PBT can clearly lie in particularly minimizing low dose areas to normal tissues.

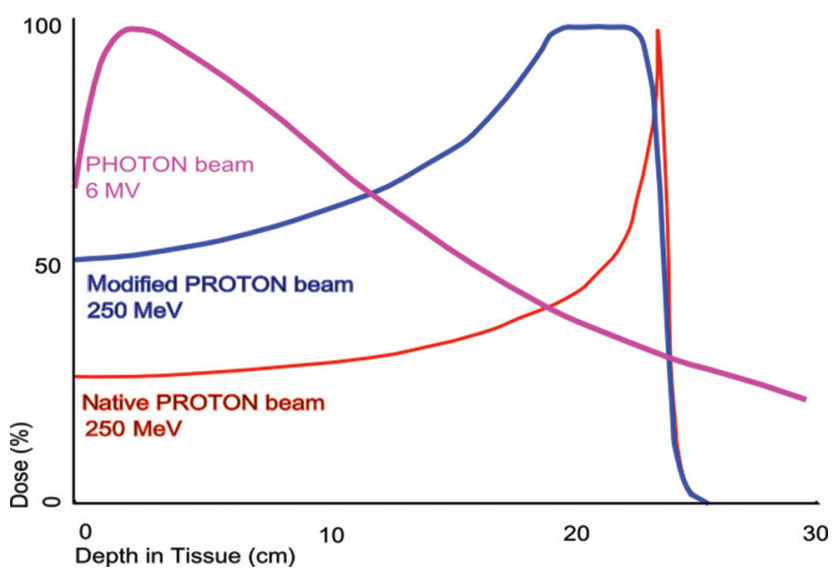

Fig. 1 The dose produced by a native and by a modified proton beam in passing through tissue, compared with the absorption of a photon or X-ray beam
The relative biological effectiveness (RBE) of protons can be defined as the ratio of the dose of a reference beam (Cobalt-60 beam) required to produce a specific effect in a biological system to the physical dose of proton radiation required to produce the same effect. Its value is not fixed, but for 70 to $250 \mathrm{MeV}$, protons range typically from 0.9 to 1.9 , with an accepted "generic" value of 1.1 in clinical proton therapy. ${ }^{10}$

The traditional PBT is riddled with uncertainties..$^{10}$ There are uncertainties in range or depth of Bragg's peak, uncertainties in biological effectiveness due to variable deposition of energy, uncertainties in dose delivery due to variations in interactions with different electron densities of tissues (e.g., electron density of bones is high and is very low for lungs), and then there are uncertainties of doses delivered due to position of mobile organs. ${ }^{10}$ However, with the current day pencil beam scanning, these are getting minimized for better treatments with lesser uncertainties.

PBT for different cancers is described below:

1. Prostate cancer: According to Cancer Research UK, ${ }^{11}$ there is an estimated 17 million new cases of cancer globally. The top four cancers occurring worldwide are lung, breast, bowel, and prostate cancer (CaP), respectively. Due to the long survival and relatively higher doses of radiation therapy, there was an interest in PBT for treatment of CaP for a possible further decrease in doses to normal tissues and better quality of life. Several dosimetric studies have demonstrated that proton therapy for prostate cancer could lower the mean dose to the rectum and bladder compared with VMAT. ${ }^{12-14}$ However, due to the close anatomic relation of bladder and rectum, there is no advantage in terms of the sparing these organs from the high dose volume. In a retrospective study, Takagi et $\mathrm{al}^{15}$ evaluated long-term outcomes of proton therapy in prostate cancer with a median follow-up of 70 months. Almost $99 \%$ of the patients received a dose of $74 \mathrm{~Gy}$. For the low-, intermediate-, high-, and very-high-risk groups, the 5-year failure-free biological recurrence was 99, 91 86 , and $66 \%$, respectively, and the 5 -year cancer-specific survival was $100,100,99$, and $95 \%$, respectively. Grade 2 or higher late gastrointestinal (GI) and genitourinary toxicities were reported to be 3.9 and $2 \%$, respectively.

Study by Kase et $\mathrm{al}^{16}$ reported results of a dosimetric study comparison between passive-scattering proton beam (PSPT) and intensity-modulated PBT (IMPT) and reported a further decrease in doses to some organs at risk (OARs).

Therefore, while there is dosimetric data of a numerical decrease in doses to surrounding areas, the high-dose areas which are a primary concern in CaP treatment do not get affected. The exact clinical value of further decrease in low-dose areas with PBT needs to be evaluated. One important aspect of studies on $\mathrm{PBT}$ in $\mathrm{CaP}^{15-17}$ is the fact that the target volume in all of them is just the prostate and surrounding tissues with the extent of depending on risk assessment. However, most studies with IMRT and IGRT include not just the prostate and surrounding tissues 
as per assessment but also include pelvic nodal stations although the same is debatable and an ongoing trial RTOG (radiation therapy oncology group) 0924 seeks to resolve the same. ${ }^{18}$ Also, an ongoing phase-III trial, The Prostate Advanced Radiation Technologies Investigating Quality of Life (PARTIQoL) comparing PBT to IMRT is ongoing to evaluate quality of life, toxicity in low, and intermediate risk CaP. ${ }^{19}$ There is also some concern with regards to the interfraction uncertainties introduced by organ filling.

The results for hypofractionated RT (HRT) reported in RTOG 0415 (70 Gy at $2.5 \mathrm{~Gy} /$ fractions), ${ }^{20}$ the CHHiP trial (60 Gy at 3Gy/fraction), ${ }^{21}$ and HYPRO study (64.6 Gy at $3.4 \mathrm{~Gy} /$ fraction $^{22}$ established the hypofractionated regimen in the treatment of carcinoma prostate. ChhiP trial reported grade 2 or worse bowel and bladder adverse events at 11.9 and $11.7 \%$ in the 60 Gy group. RTOG 0415 reported in hypofractionated arm grade-3 GI toxicity at $4.6 \%$ and grade- 3 genitourinary toxicity at $6.4 \%$. All the reported results were noninferior between conventional and hypofractionated arm. ${ }^{20-22}$

A study ${ }^{23}$ from Japan reported results on 526 patients receiving either conventional fractions for low and high risk CaP (74 Gy/37 fractions and $78 \mathrm{GY} / 39$ fractions) or hypofractionated proton therapy (HFPT) for low risk CaP (60 Gy/20 fractions and $63 \mathrm{~Gy} / 21$ fractions). The authors reported acute genitourinary toxicities greater than grade 2 , rates at $5.9 \%$ for HFPT. While these results were better for HFPT as compared with conventionally fractionated PBT, the absolute rates are quite comparable as reported in photon based hypofractionated studies. ${ }^{20-22}$ These findings were corroborated in the single arm phase- 2 study $^{24}$ on HFPT for low- and intermediate-risk CaP.

As of date, it may be wise to refer patients with low risk $\mathrm{CaP}$, very large and odd-shaped prostate or ones requiring high doses to critical areas (for e.g., a large avid perirectal lymph node) for PBT because the benefit of numerically lower doses will likely be more when the overall volumes are high or are closely related to critical structures. Hypofractionated approach in itself may not justify PBT in view of similar toxicity outcomes for photons and PBT based studies as discussed above.

2. Pediatric cancers: PBT, as we know, achieves better target coverage with lower volumes of normal tissues receiving lower doses. It can result in up to 50 to $70 \%$ reduction in the integral dose as compared with photon beam radiotherapy. ${ }^{25}$ Although there has been a significant progress in RT technology, ${ }^{26}$ the concerns about acute and long-term side effects remain. This issue assumes more importance in pediatric age-group due to the ongoing development of organs and tissues and a relatively longer life expectancy in childhood cancer survivors which makes them vulnerable to the effects of radiation on growth, intellectual development, endocrine organ function, and secondary cancer development. Therefore, the pediatric radiation dose to normal tissues should be reduced as much as possible where PBT is valuable. ${ }^{27-31}$ For this reason, PBT may be useful for the treatment of pediatrics cancers. A retrospective observational study of pediatric patients who received
PBT was reported in 2017. The 5-, 10-, and 20-year rates for grade 2 or higher late toxicities were 18,35 , and $45 \%$, respectively, and those for grade 3 or higher late toxicities were 6,17 , and $17 \%$, respectively. No malignant secondary tumors occurred within the irradiated field. ${ }^{32}$ Colaco et al showed that the tumor control rate and survival outcomes in proton therapy for treating pediatric patients diagnosed with central nervous system cancer were comparable to that of photon therapy with significant reduction in acute and late toxicities. There was also decrease in the severity of endocrine, neurological, intelligence quotient, and quality of life deficits including lesser ototoxicity. ${ }^{33,34}$

While there is no doubt that the dosimetric advantage of PBT may be best suited for pediatric age group, it is worth mentioning that critical clinical data on the longterm effectiveness, and harms associated with the use of PBT in most pediatric cancers is not available. There is still need for high quality research in this matter.

As of date, it may be wise to refer the pediatric age group for PBT except for the tumors needing small doses like Wilms' tumor.

3. Breast cancers: While adjuvant radiation therapy for breast cancer $(\mathrm{CaB})$ is important for local control and survival benefit, ${ }^{35}$ it is a known fact that long-term cardiovascular toxicities and second cancers induced by RT may counteract the survival benefit. ${ }^{36,37}$ PBT can reduce the mean dose to heart to approximately 0 to $0.5 \mathrm{~Gy}$ for left-sided breast cancer. ${ }^{38} \mathrm{~A}$ systematic review published in 2019 recommends the mean heart dose to be less than $2.5 \mathrm{~Gy} .{ }^{39} \mathrm{~A}$ recent systematic review reported doses using modern radiotherapy practices (photon based). ${ }^{40}$ They reported that the mean heart dose for left-sided breast cancer, reported by 84 studies (studies published between January 2014 and September 2017) was 3.6 Gy, compared with a review of those previously reported (5.4 Gy). They further reported that the regimens employing breathing control in any position had a significantly lower mean heart dose $(1.7 \mathrm{~Gy})$ compared with regimens without breathing control (4.5 Gy; $p<0.0001)$.

Therefore, it can be appreciated that the modern day regimens are already keeping the dose to heart at an acceptable level. Furthermore, it was reported in a recent paper that the estimated absolute risk of cardiac mortality from modern radiotherapy were approximately $1 \%$ for smokers and $0.3 \%$ for nonsmokers. ${ }^{41}$

In the recent years, relative success of accelerated partial breast irradiation (APBI) and PBI has also reduced the target volumes in breast for the radiation oncologist. This decreases the scatter dose to the heart even further. ${ }^{42,43}$

Keeping all the above in perspective, we can realize that the lower dose to heart possible with PBT may not be clinically useful for most cases.

To further determine and evaluate its cardiovascular effects, an ongoing prospective phase-II trial (NCT01758445) on patients receiving PBT for stage-II/III breast cancers has been undertaken.

However, it will be wise to consider PBT for special situations. For example, an early-stage tumor on left side 
especially in the inner/central quadrant ${ }^{44}$ or a locally advanced breast cancer on left side requiring internal mammary node radiation. ${ }^{38}$ In above-mentioned or any other situations, physical properties of PBT may help some patients, especially where modern radiotherapy techniques may not be yielding enough dosimetric benefits.

4. Hepatocellular carcinoma: Hepatocellular carcinoma (HCC) is a primary liver cancer believed to originate from primary stem cells. The treatment of choice is surgery but most patients are seen in an inoperable stage ${ }^{45}$ and then the intent of treatment becomes palliative by prolonging the liver function for as long as is possible. The problem with delivering RT to this tumor is the inherent radiosensitivity of the surrounding normal liver parenchyma. This problem is partly solved by SBRT using photon beam. Méndez Romero et $\mathrm{al}^{46}$ reported local control rates at 1 and 2 years for the whole group (which included liver metastases) were 94 and $82 \%$. A Korean group reported that higher RT doses (cumulative and per fraction) are required to achieve the same tumor control probability for larger lesions. ${ }^{47}$ This is where benefit of PBT can be explored. A dosimetric study has shown that IMPT (intensity modulated PBT) could decrease the dose delivered to organs at risk compared with IMRT-based SBRT. ${ }^{44}$ This allows us the possibility to increase the dose given to the tumor without increasing radiation-induced liver damage. ${ }^{48,49}$ Sugahara et al reported their results of a retrospective study ${ }^{50}$ on 22 patients with large HCC (median size: $11 \mathrm{~cm}$, range: $10-14 \mathrm{~cm}$ ). The patients were treated with proton therapy to a dose of $72.6 \mathrm{cGyE}$ and they demonstrated promising result with a 2-year local control (LC) of 87\%, 2-year overall survival (OS) of 36\%, 2-year progression-free survival (PFS) $24 \%$ with no grades 3 to 5 late toxicities. PBT has the potential to reduce radiation-related hepatotoxicity and allow for tumor dose escalation; however, due to the retrospective nature of the data, true survival benefit cannot be commented upon unless phase- 3 trials are undertaken. The reason for the latter is the fact that even the benefits of liver transplant are not seen beyond certain size criteria. ${ }^{51}$ The same is likely attributable to the fact that larger sized tumors are more aggressive. ${ }^{52}$ NRG-GI003 (NCT03186898) is a recently opened phase-III randomized trial that will determine if PBT will confer an OS advantage compared with photons in unresectable HCC patients.

Lastly, a recent publication from Japan examined 83 recurrent HCC cases after initial PBT. There were multiple dose-fractionation combinations for both primary and reradiation therapy (reRT; determined by tumor location within the liver), with $60 \mathrm{GyE}$ in 10 fractions and $72.6 \mathrm{GyE}$ in 22 fractions being the most common. Twoyear survival was $88 \%$, and no patient experienced grade 3 or higher adverse events. ${ }^{53}$

Currently, those patients with an upfront large tumor size that requires SBRT may be referred for IMPT based proton therapy if a sufficient dose to target is not achievable without significant damage to liver parenchyma. Also, a recurrent HCC amenable to SBRT will benefit with superior dosimetry of PBT.
5. Head and neck tumors: The most common of these are squamous cell carcinomas of head and neck (HNSCC). RT is an important treatment modality for HNSCC. PBT has shown an advantage to minimize doses to the brain stem, salivary glands, spinal cord, pharynx, and larynx in comparison with RT including newer techniques of IMRT and VMAT.5,54-57 A case-matched dosimetric analysis of 25 patients reported significant reduction to doses to OARs when IMRT was compared with IMPT. ${ }^{58}$ Ramaekers et $\mathrm{al}^{59}$ explored the cost-effectiveness of IMPT with IMRT in HNSCC using NTCP models and comparative planning studies. At 12 months, the predicted occurrence of xerostomia and dysphagia was found to be lower with IMPT (22 and 18\%) than with IMRT (44 and 23\%). However, PBT does not improve survival outcomes as compared with IMRT. ${ }^{60}$ One site which may particularly benefit with PBT is nasopharyngeal (NPC) and sinonasal carcinoma because of its special anatomic location with its close proximity to eyes, neural structures, and cranial nerves. The advantage on dosimetry for proton therapy over IMRT is a significant reduction of normal tissue volumes receiving lower radiation doses. ${ }^{61,62} \mathrm{~A}$ recent report by Lewis et $\mathrm{al}^{63}$ reported their findings on nine NPC patients who were treated with PBT and concurrent cisplatin-based chemotherapy. They reported a 2-year LC and OS was 100 and $88.9 \%$, respectively. No patients had acute grade- 4 or -5 toxicities. They reported only grade- 2 mucositis in eight patients and grade 3 in one patient. These outcomes are quite encouraging due to low-adverse events with LC and OS results that are similar to prior reports of IMRT. ${ }^{64}$

While the dosimetric advantage can be appreciated in the available dosimetric studies, there is no prospective phase-3 study toward use of PBT in head and neck cancers. A prospective randomized study between PBT and IMRT comparing the toxicity as a primary outcome is currently recruiting (NCT01893307). Therefore, to judge the need for PBT for any particular case, a model-based approach is recommended where existing NTCP models are used to choose those patients who stand to benefit in terms of avoiding toxicity, and thereby decreasing their morbidity and costs. ${ }^{64,65}$

PBT, due to its potential advantages, may result in clinically meaningful reductions in toxicities and complications in reRT for HNSCC. Many series including two prospective studies have been published in this regard to date. The results are dependent on patient selection, rates of salvage surgery, use of concomitant chemotherapy, and histology of the tumor. ReRT is not devoid of toxicity, most series report similar acute toxicity and mortality. One of the initial report regarding PBT ( $n=34)$ with recurrentoral cavity cancer utilized concurrent intra-arterial chemotherapy. ${ }^{66}$ With a retreatment median dose of 50 GyE-2-year LC was $62 \%$ and 2-year OS was $42 \%$. Although no patient experienced grade 4 and 5 toxicities, several suffered grade-3 mucositis (32\%), dermatitis (29\%), and dysphagia (35\%). One multi-institutional retrospective study $^{67}(n=92)$ evaluating reirradiated passive scatter proton therapy (PSPT) reported 1-year locoregional 
control of $75 \%$, and OS rate of $65.2 \%$. Authors reported that acute grade- 3 adverse events from PBT reRT were lower than historical IMRT reRT series. A median of 34 months elapsed between treatments. Another series from MD Anderson Cancer Center $(n=60)$ reported the rates of locoregional failure free and OS at 1 year 68.4 and $83.8 \%$, respectively. ${ }^{68}$ The median interval from initial to retreatment was 47 months. The rate of feeding tube placement was $10 \%$ and grade- 3 dysphagia and xerostomia was seen in three and two patients respectively. The authors correlated clinical target volume $>50 \mathrm{~cm}^{3}$ with greater risk of grade $>3$ toxicities. McDonald et al ${ }^{69}$ evaluated 61 patients with full-dose initial (median: 65-66 Gy). At a median 15 -month follow-up, the LRR was $20 \%$, but the 2 -year OS markedly differed from the previous study (33\% in this series). Grade-3 mucocutaneous toxicities were seen in eight patients and 1 person died of RT-induced brainstem edema leading to a fall and hematoma. On multivariate analysis, factors portending risk of local failure included presence of gross (versus microscopic) disease, and size of the gross tumor volume. ReRT dose, when analyzed as a continuous variable, independently correlated with local control. ReRT in HNSCC has always been dismal clinical scenario with varied outcomes and toxicity and PBT results do look exciting. A recent publication by multi-institution reirradiation (MIRI) collaborative group ${ }^{70}$ reported results on 412 recurrent or second primary HNSCC patients reirradiated with IMRT. They reported encouraging results with 2-year survival of up to $62 \%$ in recursive partitioning analysis (RPA) class-I patients. The overall rates of acute grade-3 toxicity were $19 \%$ and cumulative incidence of late toxicities was $14.2 \%$. This study although retrospective, reports results of a large cohort of patients and results may be considered equivalent to PBT results discussed above.

SBRT with photons is an emerging modality for treatment of recurrent HNSCC. A recent publication ${ }^{71}$ reported a 2-year survival for patients with tumor size less than $25 \mathrm{cc}$ where more than $35 \mathrm{~Gy}$ dose could be safely given. The acute grade- 4 toxicity reported in group receiving SBRT was less than $1 \%$.

Therefore, it can be appreciated that survival outcomes for recurrent HNSCC patients depends upon time to recurrence, operability, and size or volume and presence of organ dysfunction. Toxicity reports for non-PBT therapies of IMRT and SBRT are not dismal and are may be even equivalent.

However, the heterogeneity within recurrent HNSCC cases forbids us to generalize and recommend any one particular modality (IMRT, SBRT, or PBT) for RT. The decision may be empirically based on closeness to critical structures. Ideally MIRI RPA class I must receive fractionated RT (preferably with chemotherapy) $)^{70}$ and if such a planning target volume (PTV) is reasonably close to critical structures (especially neural), then the same may not be possible with IMRT and PBT may be preferable. Regarding MIRI RPA class II where PTV that may be too close to the critical structures, and it is not possible to consider the patient for fractionated RT, then we can go for SBRT if the volume is less than $35 \mathrm{cc}$ or for fractionated PBT with concurrent chemotherapy.

ReRT with PBT in this scenario has a high potential for minimizing toxicities and as systematic randomized study with suitable RPA class groups with QOL analysis is much needed.

6. Skull base chordoma: Chordoma is a rare, locally aggressive, slow-growing primary bone tumor. It occurs typically on either end of the notochord, which during development transforms into the sacrum and the skull base. Traditionally, they have been addressed with surgery, but the extent of surgical resection at the skull base is often limited by the surrounding critical neurovascular structures. Subtotal resections are, therefore, common and residual tumors are meant to be treated by radiation therapy ${ }^{72}$ Chordoma cells are considered relatively radioresistant when treated with conventional fractionated external beam protocols, and therefore, high doses are warranted. ${ }^{73}$ Due to its proximity to neural tissue and the higher dose requirement, PBT is evolving as the postoperative treatment of choice. ${ }^{74,75}$ A review reported results of 416 cases of advanced or incompletely resected tumors comparing photons to PBT, PBT showed better results with the best long-term (10 years) outcomes with relatively few significant complications considering the high doses delivered with this therapeutic modality. ${ }^{76}$ Another review conducted in 2018 concluded that despite advances in proton therapy, recurrence rates in skull base chordoma remain high. ${ }^{77}$ Large case series demonstrated that unfavorable tumor control was often linked to high residual volumes $\left(>23 \mathrm{~cm}^{3}\right)$ and brainstem involvement. ${ }^{78-80}$ Authors recommended that due to the radioresistant behavior of this neoplasm, a delivery of a dose of at least $74 \mathrm{~Gy}$ (RBE) to the tumor bed and other sites of gross disease is recommended. As an alternative to PBT, modern technology in conformal photon irradiation can offer encouraging control rates. In a recent report on 24 patients treated with IMRT and IGRT to a median dose of $76 \mathrm{~Gy}$, the overall 5-year loco-regional control (LRC) was $65.3 \% .^{81}$ Larger tumor volume of tumor (>60 cc)and reirradiation were significant prognostic factors predict inferior survival. ${ }^{82}$

Due to the close proximity to brain stem, it is very challenging to treat these patients with an ideal dose of 76 cobal grey equivalent (CGE) even with modern technology for photon beam RT therefore PBT seems to be a very exciting clinical tool. However, due to the very same reason of close proximity with the dose and range uncertainties of PBT, it may not always be possible to control dose to brain stem so easily. This is due to the fact that the dose needed to treat chordomas and chondrosarcomas is a high dose of greater than 70 CGE and the dose tolerable to brain stem is just a maximum voxel dose of 60Gy. This steep fall-off may not always be possible due to their close proximity. However, PBT may still be our best hope for better dosimetry in this critical location. Also, prospective trials are currently being conducted to compare the biological effectiveness and safety of carbon-ion therapy with PT for skull base chordomas. ${ }^{74}$ 
7. GI tumors: PBT has been explored in gastrointestinal tract tumors with the aim to reduce toxicities. In a study from Florida, 22 patients were treated with proton therapy and concomitant capecitabine (1,000 mg per orally twice daily) for resected $(n=5)$; marginally resectable $(n=5)$ and unresectable/inoperable $(n=12)$ biopsy-proven pancreatic and ampullary adenocarcinoma. ${ }^{83}$ Proton doses ranged from 50.40 to 59.40 CGE. Median follow-up was 11 months. No patient demonstrated any grade- 3 toxicity during treatment or during the follow-up period and grade-2 GI toxicity was eliminated $(p=0.0009$ ) in patients treated with plans that avoided anterior and left lateral fields which were associated with reduced small bowel and gastric exposure. Mizumoto et al reported 51 patients with esophageal cancer who were treated between 1985 and 2005 using proton beams with or without photons. ${ }^{84}$ There was no treatment interruption and the overall 5 -year actuarial survival rate was $21.1 \%$ and the median survival time was 20.5 months. The 5 -year local control rate for all 51 patients was $38.0 \%$ and the median local control time was 25.5 months. Studies have evaluated role PBT in recurrent GI neoplasms. Ono et al reported the results of multicenter retrospective study in Japan with 202 (including 90 inoperable) esophageal cancer patients. ${ }^{85}$ The 3 -year and 5 -year OS was 66.7 and $56.3 \%$, respectively. The 5 -year LRC was $64.4 \%$. This study suggests that PBT for esophageal cancer was not inferior in efficacy and had lower rates of toxicities in comparison to photon radiotherapy.

These studies report encouraging results with PBT for GI cancers. Though lower grade-2 toxicities are inspiring, a randomized study with an assessment of quality of life shall be very helpful in recommending PBT for these patients.

8. Lung cancers: PBT is associated with more uncertainty due to finite range of proton beam and motion for thoracic cancers. It is less forgiving in moving lung cancer due to the Bragg peak's sensitivity to motion, anatomy, and density changes. Although PBT is improving rapidly as more research is performed with the implementation of four-dimensional computed tomography (CT)-based motion management and IMPT. ${ }^{86}$ In the middle of 1990s, the Loma Linda University lung cancer program developed techniques for managing early-stage NSCLC using PBT, they intensified the dose to $70 \mathrm{~Gy}$ (RBE) over 10 fractions. ${ }^{87}$ This led numerous proton centers in Japan and United States to consider hypofractionated and accelerated PBT to manage stage-I NSCLC. ${ }^{88-90}$

A study compared PSPT, IMPT, and photon-based radiotherapy to deliver SABR, $50 \mathrm{~Gy}$ in four fractions in centrally located stage-I NSCLC, and reported significant reduction of radiation doses to critical structures. ${ }^{91}$ Other studies reported that the proton-based SBRT plans delivered a lower radiation dose to lungs, esophagus, bronchial tree, and spinal cord. ${ }^{92,93}$ PBT was noted to deliver a slightly higher radiation dose to the skin and chest wall when less than three beams were used but was overcome by use of a greater number of proton beam fields or arc therapy. As expected, PSPT is not better for dose reduction for esophagus, lung receiving $20 \mathrm{~Gy}$, or higher. However, optimized PSPT better controls lung V5 and spares better than contralateral lung, spinal cord, heart, and integral dose compared with photon plans. ${ }^{94,95}$

A major limitation to proton therapy in early NSCLC was the lack of volumetric image guidance, thereby requiring slightly larger PTV margins for uncertainty.

While the doses to OARs is lower with PBT (especially with IMPT) the clinical outcomes remain similar and therefore PBT may be better suited for patients where lowering the volumes of normal tissues that receive the low doses shall be clinically meaningful. For example, patients with larger early stage tumors, smaller overall lung volume, tumors located more centrally, or those that are close to the brachial plexus. Also, like always PBT may be considered for patients who don't meet dosimetric constraints with photon based SBRT.

A phase-II study of 44 patients with stage-III NSCLC who received 74 CGE via conventional fractionation (two CGE per fraction) with weekly concurrent carboplatin and paclitaxel, reported no grade- 4 or -5 toxicities, and minimal grade- 3 toxicities. ${ }^{96}$ The median survival was 29.4 months, as compared with 20.3 months of 74 Gy arm in RTOG 0617. Two similarly designed studies that included fewer patients, demonstrated equally excellent outcomes. ${ }^{97,98}$ An ongoing phase-III study RTOG 1308 comparing IMRT to proton therapy in stage-III NSCLC seeks to evaluate OS as its primary objective. A randomized study to compare IMRT with IMPT using an integrated boost approach to boost GTV to $72 \mathrm{~Gy}$, while keeping PTV dose to $60 \mathrm{~Gy}$ with concurrent chemotherapy is ongoing. Two studies are currently exploring hypofractionation with concurrent chemotherapy including a single institutional study and a multicenter study being done through the Proton Collaborative Group (PCG).

Due to these controversies of range uncertainty and motion management, there is much debate in the oncology community about which lung cancer patients benefit significantly from proton therapy. PBT could be the answer to successful dose escalation especially in large treatment volumes encountered in stage three NSCLC. Often such patients are taken up for neoadjuvant chemotherapy to no particular survival benefit with a higher toxicity. ${ }^{99}$ PBT should be particularly beneficial to patients who have poor pulmonary function, cardiovascular disease, recurrent disease, or other individuals at high-risk to develop severe side effects in general (such as the elderly).

9. Reirradiation with PBT: Locoregional recurrences within the previously irradiated fields are common even with the advancement in radiation techniques, newer systemic therapies, and improved surgical techniques. Reirradiation is challenging in effective and safe dose delivery and the dosimetric advantage of proton therapy makes it an exciting option to reirradiate with minimal toxicity to normal tissues.

In a study by Dionisi et al, ${ }^{100} 17$ recurrent NPC were treated by PBT with median reirradiation dose of $60 \mathrm{~Gy}$ RBE (range: 30.6-66) with median follow-up of 10 months. $53 \%$ of patient underwent concomitant chemotherapy. 
No adverse grade- 3 acute toxicity seen and late grade- 3 toxicity seen in $23.5 \%$, with hearing impairment in $17.6 \%$. Two patients developed grade- 2 soft tissue necrosis and grade- 5 carotid blowout occurred in one patient. Overall survival was $54.4 \%$ and local control was $66.6 \%$.

Retrospective study on PBT with IMPT reirradiation of thoracic malignancies with definitive intent was done by Ho et al. The study included 27 patients. 93\% of patients received IMPT for thoracic cancer recurrence and 7\% patients received sequential PBT after a course of SABR. A median dose of 66 Eq. D2 Gy (range, 43.2-84 Gy). The median time to reirradiation was 29.5 months and median follow-up for all patients was 11.2 months (25.9 surviving patients). The 1-year LRFS and PFS were 84 and 76\%, respectively. Late grade-3 pulmonary toxicity developed in two patients and grade 4-5 toxicities was not seen in any patient. ${ }^{101}$

Fifty patients (2011-2016) who received PBT reirradiation for breast cancer got registered in the PCG. ${ }^{102}$ Median follow-up of 12.7 months (range: 0-41.8) and $60 \mathrm{~Gy}$ (range: 10-96.7) of median prior RT dose were given. Median reirradiation dose was 55.1 Gy (range: 45.1-6.3 Gy) and 110.6 Gy (range: 70.6-156.8) of median cumulative dose. Median interval between RT courses was 103.8 months (range: $5.5-430.8$ ). A total of $84 \%$ regional nodes was included in reRT in which $66 \%$ were internal mammary node (IMN). At 1 year, LRFS was 93\%, and OS was $97 \%$. Grade- 3 events occurred in patient with body mass index (BMI) more than 30, IMN reirradiation and bilateral breast cancer with acceptable toxicity in spite of median cumulative dose exceeding $110 \mathrm{~Gy}$.

Fifteen patients with isolated, locally recurrent pancreatic cancer were treated with PBT were analyzed from 2010 to 2015. Median follow-up was 15.7 months (range: 2-48 months). Concurrent chemotherapy was received by $67 \%$ of the patients and median PRT dose was $59.4 \mathrm{~Gy}$ (range: 37.5-59.4 Gy) with median time interval of 26.7 months (range: 7-461.3 months). Acute $\geq$ grade-3 toxicities was seen in $13 \%$ of patients. One-year OS was $67 \%$ and "in-field" failure-free survival at 1 year was $87 \%$. LPFS and DMFS at 1 year was 72 and $64 \%$, respectively, with a higher median survival found with concurrent chemotherapy. ${ }^{103}$

Fifteen patients of recurrent rectal and anal cancer were analyzed using a hyperfractionated or accelerated reRT by PBT from 2007 to 2017 with a median prior pelvic RT dose of $50.4 \mathrm{~Gy}$ (range: $25-80 \mathrm{~Gy}$ ) and median time interval of 4.7 years (range: $1.0-36.1$ years). Total reRT dose was 39 to $45 \mathrm{~Gy}$ (RBE) along with concurrent chemotherapy to all patients. As compared with photon therapy PBT achieved lower mean pelvic bone marrow dose and acute or late grade- 4 toxicity was not seen in any patients. ${ }^{104}$

In a prospective study, 14 patients were enrolled for thoracic reirradition between 2010 and 2014. All 14 patients had history of thoracic radiation and newly diagnosed or locally recurrent esophageal carcinoma. All patients received proton beam reirradiation. The median reirradiation dose was 54.0 Gy (RBE; range: 50.4-61.2 Gy) and median cumulative dose was $109.8 \mathrm{~Gy}$ (range: 76-129.4 Gy). Concurrent chemotherapy was received by eleven patients. The median interval between radiation courses was 32 months (range: 10-307 months) and the median follow-up was 10 months (range: 2-25 months). This study ${ }^{105}$ showed comparable acute and late toxicity with good local control with maximum radiation related grade-2 toxicity of $64 \%$.

The potential advantage of PBT in reirradiation of meningioma is due to lower integral brain dose. Sixteen patients were retrospectively analyzed who received prior median dose of $54 \mathrm{~Gy}$ (range: 13-65.5 Gy). Median time from prior RT to PBT, reRT was 5.8 years (range: 0.718.7 years) and median PBRT dose was $60 \mathrm{~Gy}$ (RBE; range: 30-66.6 Gy). Median follow-up was 18.8 months. Patients with initially grade-I tumors and longer interval between prior RT and PBRT had improved PFS. Late grade- 3 toxicity was $31 \%$ and $13 \%$ developed radionecrosis with good intracranial control. ${ }^{106}$

The results with reirradiation for HNSCC were discussed in the relevant section. The other studies reviewed above report reasonably good outcomes with toxicities that are not unacceptably high. The reRT is generally the last option as it is generally advised only when the no other options are beneficial or feasible for a patient. In such a scenario, PBT results are indeed encouraging though high quality phase-3 data with a focus on QOL shall help oncologists make data-driven choices for their patients.

\section{Conclusion and Future Directions}

The physical properties of protons provide PBT with unique dosimetric advantages with only few disadvantages. PBT therefore remains a promising treatment which has already shown many positive results. However, the most important benefit that comes is in reduction of low dose volumes which means that the volumes of normal tissues receiving less than $50 \%$ of prescribed dose are lesser for PBT but not necessarily the high-dose volumes. ${ }^{55}$ Therefore, we cannot always presume superior outcomes with PBT even though it may be intuitive to do so. It is for this reason that we should encourage phase- 3 trials to compare the modalities so that robust data can be obtained for a more scientifically sound decision making for our patients. It is also important to examine longterm clinical outcomes with PBT to truly understand the benefits of PBT.

However, radiation oncology is a branch where tools and techniques always makes a difference and therefore there must be a careful consideration for PBT treatment for pediatric cases and for complex cases in adults where dosimetry may be unsuitable to the planned treatment in terms of coverage and sparing of normal tissues.

\section{Conflict of Interest}

None declared. 


\section{References}

1 Garibaldi C, Jereczek-Fossa BA, Marvaso G, et al. Recent advances in radiation oncology. Ecancermedicalscience 2017;11:785

2 Baumann M, Krause M, Overgaard J, et al. Radiation oncology in the era of precision medicine. Nat Rev Cancer 2016;16(4):234-249Review

3 Schiller KC, Habl G, Combs SE. Protons, photons, and the prostate - is there emerging evidence in the ongoing discussion on particle therapy for the treatment of prostate cancer? Front Oncol 2016;6:8

4 Ho ESQ Barrett SA, Mullaney LM. A review of dosimetric and toxicity modeling of proton versus photon craniospinal irradiation for pediatrics medulloblastoma. Acta Oncol 2017;56(8):1031-1042

5 Kandula S, Zhu X, Garden AS, et al. Spot-scanning beam proton therapy vs intensity-modulated radiation therapy for ipsilateral head and neck malignancies: a treatment planning comparison. Med Dosim 2013;38(4):390-394

6 Wilson RR. Radiological use of fast protons. Radiology 1946;47(5):487-491

7 Lawrence JH, Tobias CA, Born JL, et al. Pituitary irradiation with high-energy proton beams: a preliminary report. Cancer Res 1958;18(2):121-134

8 Tian X, Liu K, Hou Y, Cheng J, Zhang J. The evolution of proton beam therapy: Current and future status. Mol Clin Oncol 2018;8(1):15-21

9 Bragg WH, Kleeman R. On theoparticles of radium, and their loss of range in passing through various atoms and molecules. Philos Mag 1905;10:318-340

10 Engelsman M, Schwarz M, Dong L. Physics controversies in proton therapy. Semin Radiat Oncol 2013;23(2):88-96

11 Research UK. Worldwide Cancer Statistics. Available at: https://www.cancerresearchuk.org/health-professional/cancer-statistics/worldwide-cancer. Accessed on April 13, 2020

12 Deville C Jr., Jain A, Hwang WT, et al. Initial report of the genitourinary and gastrointestinal toxicity of post-prostatectomy proton therapy for prostate cancer patients undergoing adjuvant or salvage radiotherapy. Acta Oncol 2018;57(11): 1506-1514

13 Goddard LC, Brodin NP, Bodner WR, Garg MK, Tomé WA. Comparing photon and proton-based hypofractioned SBRT for prostate cancer accounting for robustness and realistic treatment deliverability. Br J Radiol 2018;91(1085):20180010

14 Fellin F, Azzeroni R, Maggio A, et al. Helical tomotherapy and intensity modulated proton therapy in the treatment of dominant intraprostatic lesion: a treament planning comparison. Radiother Oncol 2013;107(2):207-212

15 Takagi M, Demizu Y, Terashima K, et al. Long-term outcomes in patients treated with proton therapy for localized prostate cancer. Cancer Med 2017;6(10):2234-2243

16 Kase Y, Yamashita $\mathrm{H}$, Fuji $\mathrm{H}$, et al. A treatment planning comparison of passive-scattering and intensity-modulated proton therapy for typical tumor sites. J Radiat Res (Tokyo) 2012;53(2):272-280

17 Hoshina RM, Matsuura T, Umegaki K, Shimizu S. A Literature Review of Proton Beam Therapy for Prostate Cancer in Japan. J Clin Med 2019;8(1):48

18 Androgen-deprivation therapy and radiation therapy in treating patients with prostate cancer. Available at: https://clinicaltrials.gov/ct2/show/NCT01368588. Accessed May 27, 2020

19 Proton Therapy vs. IMRT for Low or Intermediate Risk Prostate Cancer (PARTIQoL). Available at: https://clinicaltrials.gov/ct2/ show/NCT01617161. Accessed May 27, 2020

20 Lee WR, Dignam JJ, Amin MB, et al. Randomized phase III noninferiority study comparing two radiotherapy fractionation schedules in patients with low-risk prostate cancer. J Clin Oncol 2016;34(20):2325-2332

21 Dearnaley D, Syndikus I, Mossop H, et al; CHHiP Investigators. Conventional versus hypofractionated high-dose intensity-modulated radiotherapy for prostate cancer: 5-year outcomes of the randomised, non-inferiority, phase $3 \mathrm{CHHiP}$ trial. Lancet Oncol 2016;17(8):1047-1060

22 Incrocci L, Wortel RC, Alemayehu WG, et al. Hypofractionated versus conventionally fractionated radiotherapy for patients with localised prostate cancer (HYPRO): final efficacy results from a randomised, multicentre, open-label, phase 3 trial. Lancet Oncol 2016;17(8):1061-1069

23 Nakajima K, Iwata H, Ogino $\mathrm{H}$, et al. Acute toxicity of imageguided hypofractionated proton therapy for localized prostate cancer. Int J Clin Oncol 2018;23(2):353-360

24 Grewal AS, Schonewolf C, Min EJ, et al. Four-year outcomes from a prospective phase II clinical trial of moderately hypofractionated proton therapy for localized prostate cancer. Int J Radiat Oncol Biol Phys 2019;105(4):713-722

25 Lomax AJ, Bortfeld T, Goitein G, et al. A treatment planning inter-comparison of proton and intensity modulated photon radiotherapy. Radiother Oncol 1999;51(3):257-271

26 Johnstone PA, McMullen KP, Buchsbaum JC, Douglas JG, Helft P. Pediatric CSI: are protons the only ethical approach? Int J Radiat Oncol Biol Phys 2013;87(2):228-230

27 Sethi RV, Shih HA, Yeap BY, et al. Second nonocular tumors among survivors of retinoblastoma treated with contemporary photon and proton radiotherapy. Cancer 2014;120(1):126-133

28 Taddei PJ, Mirkovic D, Fontenot JD, et al. Stray radiation dose and second cancer risk for a pediatric patient receiving craniospinal irradiation with proton beams. Phys Med Biol 2009;54(8):2259-2275

29 Merchant TE, Kiehna EN, Li C, et al. Modeling radiation dosimetry to predict cognitive outcomes in pediatric patients with CNS embryonal tumors including medulloblastoma. Int J Radiat Oncol Biol Phys 2006;65(1):210-221

30 Miralbell R, Lomax A, Cella L, Schneider U. Potential reduction of the incidence of radiation-induced second cancers by using proton beams in the treatment of pediatric tumors. Int J Radiat Oncol Biol Phys 2002;54(3):824-829

31 Athar BS, Paganetti H. Comparison of second cancer risk due to out-of-field doses from 6-MV IMRT and proton therapy based on 6 pediatric patient treatment plans. Radiother Oncol 2011;98(1):87-92

32 Mizumoto M, Murayama S, Akimoto T, et al. Long-term follow-up after proton beam therapy for pediatric tumors: a Japanese national survey. Cancer Sci 2017;108(3):444-447

33 Colaco RJ, Hoppe BS, Flampouri S, et al. Rectal toxicity after proton therapy for prostate cancer: an analysis of outcomes of prospective studies conducted at the university of Florida Proton Therapy Institute. Int J Radiat Oncol Biol Phys 2015;91(1):172-181

34 Leroy R, Benahmed N, Hulstaert F. Van Damme N, De Ruysscher D. Proton therapy in children: a systematic review of clinical effectiveness in 15 pediatric cancers. Int J Radiat Oncol Biol Phys 2016;95(1):267-278

35 Darby S, McGale P, Correa C, et al; Early Breast Cancer Trialists' Collaborative Group (EBCTCG). Effect of radiotherapy after breast-conserving surgery on 10-year recurrence and 15-year breast cancer death: meta-analysis of individual patient data for 10,801 women in 17 randomised trials. Lancet 2011;378(9804):1707-1716

36 Darby SC, Ewertz M, McGale P, et al. Risk of ischemic heart disease in women after radiotherapy for breast cancer. $\mathrm{N}$ Engl J Med 2013;368(11):987-998

37 Berrington de Gonzalez A, Curtis RE, Kry SF, et al. Proportion of second cancers attributable to radiotherapy treatment in 
adults: a cohort study in the US SEER cancer registries. Lancet Oncol 2011;12(4):353-360

38 Dasu A, Flejmer AM, Edvardsson A, Witt Nyström P. Normal tissue sparing potential of scanned proton beams with and without respiratory gating for the treatment of internal mammary nodes in breast cancer radiotherapy. Phys Med 2018;52:81-85

39 Piroth MD, Baumann R, Budach W, et al. Heart toxicity from breast cancer radiotherapy : Current findings, assessment, and prevention. Strahlenther Onkol 2019;195(1):1-12

40 Drost L, Yee C, Lam H, et al. A Systematic Review of Heart Dose in Breast Radiotherapy. Clin Breast Cancer 2018;18(5):e819-e824

41 Taylor C, Correa C, Duane FK, et al; Early Breast Cancer Trialists' Collaborative Group. Estimating the risks of breast cancer radiotherapy: evidence from modern radiation doses to the lungs and heart and from previous randomized trials. J Clin Oncol 2017;35(15):1641-1649

42 Moran JM, Ben-David MA, Marsh RB, et al. Accelerated partial breast irradiation: what is dosimetric effect of advanced technology approaches? Int J Radiat Oncol Biol Phys 2009;75(1):294-301

43 Sato K, Mizuno Y, Fuchikami H, et al. Comparison of radiation dose to the left anterior descending artery by whole and partial breast irradiation in breast cancer patients. J Contemp Brachytherapy 2015;7(1):23-28

44 Kumar R, Saraswat MK, Sharma BC, Sakhuja P, Sarin SK. Characteristics of hepatocellular carcinoma in India: a retrospective analysis of 191 cases. QJM 2008;101(6):479-485

45 Méndez Romero A, Wunderink W, Hussain SM, et al. Stereotactic body radiation therapy for primary and metastatic liver tumors: A single institution phase i-ii study. Acta Oncol 2006;45(7):831-837

46 Jang WI, Kim MS, Bae SH, et al. High-dose stereotactic body radiotherapy correlates increased local control and overall survival in patients with inoperable hepatocellular carcinoma. Radiat Oncol 2013;8:250

47 Cozzi L, Comito T, Fogliata A, Franzese C, Tomatis S, Scorsetti M. Critical appraisal of the potential role of intensity modulated proton therapy in the hypofractionated treatment of advanced hepatocellular carcinoma. PLoS One 2018;13(8):e0201992

48 Toramatsu C, Katoh N, Shimizu S, et al. What is the appropriate size criterion for proton radiotherapy for hepatocellular carcinoma? A dosimetric comparison of spot-scanning proton therapy versus intensity-modulated radiation therapy. Radiat Oncol 2013;8:48

49 Yoo GS, Yu JI, Park HC. Proton therapy for hepatocellular carcinoma: Current knowledges and future perspectives. World J Gastroenterol 2018;24(28):3090-3100

50 Sugahara S, Oshiro Y, Nakayama H, et al. Proton beam therapy for large hepatocellular carcinoma. Int J Radiat Oncol Biol Phys 2010;76(2):460-466

51 Zhu Z. Milan criteria and its expansions in liver transplantation for hepatocellular carcinoma. Hepatobiliary Surg Nutr 2016;5(6):498-502

52 Wu G, Wu J, Wang B, Zhu X, Shi X, Ding Y. Importance of tumor size at diagnosis as a prognostic factor for hepatocellular carcinoma survival: a population-based study. Cancer Manag Res 2018;10:4401-4410

53 Oshiro Y, Mizumoto M, Okumura T, et al. Analysis of repeated proton beam therapy for patients with hepatocellular carcinoma. Radiother Oncol 2017;123(2):240-245

54 Stuschke M, Kaiser A, Abu-Jawad J, Pöttgen C, Levegrün S, Farr J. Re-irradiation of recurrent head and neck carcinomas: comparison of robust intensity modulated proton therapy treatment plans with helical tomotherapy. Radiat Oncol 2013;8:93

55 Jakobi A, Bandurska-Luque A, Stützer K, et al. Identification of patient benefit from proton therapy for advanced head and neck cancer patients based on individual and subgroup normal tissue complication probability analysis. Int J Radiat Oncol Biol Phys 2015;92(5):1165-1174

56 van der Laan HP, van de Water TA, van Herpt HE, et al; Rococo cooperative group. The potential of intensity-modulated proton radiotherapy to reduce swallowing dysfunction in the treatment of head and neck cancer: A planning comparative study. Acta Oncol 2013;52(3):561-569

57 Wang LW, Liu YH, Chou FI, Jiang SH. Clinical trials for treating recurrent head and neck cancer with boron neutron capture therapy using the Tsing-Hua Open Pool Reactor. Cancer Commun (Lond) 2018;38(1):37

58 Holliday EB, Kocak-Uzel E, Feng L, et al. Dosimetric advantages of intensity-modulated proton therapy for oropharyngeal cancer compared with intensity-modulated radiation: A casematched control analysis. Med Dosim 2016;41(3):189-194

59 Ramaekers BLT, Grutters JPC, Pijls-Johannesma M, Lambin P, Joore MA, Langendijk JA. Protons in head-and-neck cancer: bridging the gap of evidence. Int J Radiat Oncol Biol Phys 2013;85(5):1282-1288

60 Waddle MR, Heckman M, Diehl NN, et al. Survival after proton and photon radiation therapy in patients with head and neck cancers: a study of the national cancer database. Int J Radiat Oncol Biol Phys 2018;100:1330

61 Blanchard P, Gunn GB, Lin A, Foote RL, Lee NY, Frank SJ. Proton therapy for head and neck cancers. Semin Radiat Oncol 2018;28(1):53-63

62 Cheng Q, Roelofs E, Ramaekers BLT, et al. Development and evaluation of an online three-level proton vs photon decision support prototype for head and neck cancer - Comparison of dose, toxicity and cost-effectiveness. Radiother Oncol 2016;118(2):281-285

63 Lewis GD, Holliday EB, Kocak-Uzel E, et al. Intensity-modulated proton therapy for nasopharyngeal carcinoma: Decreased radiation dose to normal structures and encouraging clinical outcomes. Head Neck 2016;38(suppl 1) :E1886-E1895

64 Widesott L, Pierelli A, Fiorino C, et al. Intensity-modulated proton therapy versus helical tomotherapy in nasopharynx cancer: planning comparison and NTCP evaluation. Int J Radiat Oncol Biol Phys 2008;72(2):589-596

65 Roelofs E, Engelsman M, Rasch C, et al; ROCOCO Consortium. Results of a multicentric in silico clinical trial (ROCOCO): comparing radiotherapy with photons and protons for non-small cell lung cancer. J Thorac Oncol 2012;7(1):165-176

66 Hayashi Y, Nakamura T, Mitsudo K, et al. Re-irradiation using proton beam therapy combined with weekly intra-arterial chemotherapy for recurrent oral cancer. Asia Pac J Clin Oncol 2017;13(5):e394-e401

67 Romesser PB, Cahlon O, Scher ED, et al. proton beam reirradiation for recurrent head and neck cancer: multi-institutional report on feasibility and early outcomes. Int J Radiat Oncol Biol Phys 2016;95(1):386-395

68 Phan J, Sio TT, Nguyen TP, et al. Reirradiation of head and neck cancers with proton therapy: outcomes and analyses. Int J Radiat Oncol Biol Phys 2016;96(1):30-41

69 McDonald MW, Zolali-Meybodi O, Lehnert SJ, et al. Reirradiation of recurrent and second primary head and neck cancer with proton therapy. Int J Radiat Oncol Biol Phys 2016;96(4):808-819

70 Ward MC, Riaz N, Caudell JJ, et al; Multi-Institution Reirradiation (MIRI) Collaborative. Refining patient selection for reirradiation of head and neck squamous carcinoma in the IMRT Era: a multi-institution cohort study by the miri collaborative. Int J Radiat Oncol Biol Phys 2018;100(3):586-594

71 Vargo JA, Ward MC, Caudell JJ, et al. A multi-institutional comparison of SBRT and imrt for definitive reirradiation of recurrent or second primary head and neck cancer. Int J Radiat Oncol Biol Phys 2018;100(3):595-605 
72 Fagundes MA, Hug EB, Liebsch NJ, Daly W, Efird J, Munzenrider JE. Radiation therapy for chordomas of the base of skull and cervical spine: patterns of failure and outcome after relapse. Int J Radiat Oncol Biol Phys 1995;33(3):579-584

73 Stacchiotti S, Sommer J; Chordoma Global Consensus Group. Building a global consensus approach to chordoma: a position paper from the medical and patient community. Lancet Oncol 2015;16(2):e71-e83

74 Nikoghosyan AV, Karapanagiotou-Schenkel I, Münter MW, Jensen AD, Combs SE, Debus J. Randomised trial of proton vs. carbon ion radiation therapy in patients with chordoma of the skull base, clinical phase III study HIT-1-Study. BMC Cancer 2010;10:607

75 Sahgal A, Chan MW, Atenafu EG, et al. Image-guided, intensity-modulated radiation therapy (IG-IMRT) for skull base chordoma and chondrosarcoma: preliminary outcomes. Neuro-oncol 2015;17(6):889-894

76 Munzenrider JE, Liebsch NJ. Proton therapy for tumors of the skull base. Strahlenther Onkol 1999;175(suppl 2) :57-63

77 Alahmari M, Temel Y. Skull base chordoma treated with proton therapy: A systematic review. Surg Neurol Int 2019;10:96

78 Hug EB, Loredo LN, Slater JD, et al. Proton radiation therapy for chordomas and chondrosarcomas of the skull base. J Neurosurg 1999;91(3):432-439

79 Noël G, Feuvret L, Calugaru V, et al. Chordomas of the base of the skull and upper cervical spine. One hundred patients irradiated by a 3D conformal technique combining photon and proton beams. Acta Oncol 2005;44(7):700-708

80 Weber DC, Malyapa R, Albertini F, et al. Long term outcomes of patients with skull-base low-grade chondrosarcoma and chordoma patients treated with pencil beam scanning proton therapy. Radiother Oncol 2016;120(1):169-174

81 Amichetti M, Amelio D, Minniti G. Radiosurgery with photons or protons for benign and malignant tumours of the skull base: a review. Radiat Oncol 2012;7:210

82 Guan X, Gao J, Hu J, et al. The preliminary results of proton and carbon ion therapy for chordoma and chondrosarcoma of the skull base and cervical spine. Radiat Oncol 2019;14(1):206

83 Nichols RC Jr., George TJ, Zaiden RA Jr., et al. Proton therapy with concomitant capecitabine for pancreatic and ampullary cancers is associated with a low incidence of gastrointestinal toxicity. Acta Oncol 2013;52(3):498-505

84 Mizumoto M, Shinji Sugahara, Hidetsugu Nakayama, et al. Clinical results of proton-beam therapy for locoregionally advanced esophageal cancer. Strahlenther Onkol 2010;186(9): 482-488

85 Ono $\mathrm{T}$, Wada $\mathrm{H}$, Ishikawa $\mathrm{H}$, Tamamura $\mathrm{H}$, Tokumaru $\mathrm{S}$. Clinical results of proton beam therapy for esophageal cancer: multicenter retrospective study in japan. Cancers (Basel) 2019;11(7):993

86 Chang JY, Jabbour SK, De Ruysscher D, et al; International Particle Therapy Co-operative Group (PTCOG) Thoracic Subcommittee. Consensus statement on proton therapy in early-stage and locally advanced non-small cell lung cancer. Int J Radiat Oncol Biol Phys 2016;95(1):505-516

87 Bush DA, Slater JD, Bonnet R, et al. Proton-beam radiotherapy for early-stage lung cancer. Chest 1999;116(5):1313-1319

88 Westover KD, Seco J, Adams JA, et al. Proton SBRT for medically inoperable stage I NSCLC. J Thorac Oncol 2012;7(6):1021-1025

89 Kanemoto A, Okumura T, Ishikawa $\mathrm{H}$, et al. Outcomes and prognostic factors for recurrence after high-dose proton beam therapy for centrally and peripherally located stage I nonsmall-cell lung cancer. Clin Lung Cancer 2014;15(2):e7-e12
90 Iwata H, Murakami M, Demizu Y, et al. High-dose proton therapy and carbon-ion therapy for stage I nonsmall cell lung cancer. Cancer 2010;116(10):2476-2485

91 Register SP, Zhang X, Mohan R, Chang JY. Proton stereotactic body radiation therapy for clinically challenging cases of centrally and superiorly located stage I non-small-cell lung cancer. Int J Radiat Oncol Biol Phys 2011;80(4):1015-1022

92 Hoppe BS, Huh S, Flampouri S, et al. Double-scattered proton-based stereotactic body radiotherapy for stage I lung cancer: a dosimetric comparison with photon-based stereotactic body radiotherapy. Radiother Oncol 2010;97(3):425-430

93 Macdonald OK, Kruse JJ, Miller JM, et al. Proton beam radiotherapy versus three-dimensional conformal stereotactic body radiotherapy in primary peripheral, early-stage non-small-cell lung carcinoma: a comparative dosimetric analysis. Int J Radiat Oncol Biol Phys 2009;75(3):950-958

94 Albertini F, Bolsi A, Lomax AJ, Rutz HP, Timmerman B, Goitein G. Sensitivity of intensity modulated proton therapy plans to changes in patient weight. Radiother Oncol 2008;86(2):187-194

95 Kadoya N, Obata Y, Kato T, et al. Dose-volume comparison of proton radiotherapy and stereotactic body radiotherapy for non-small-cell lung cancer. Int J Radiat Oncol Biol Phys 2011;79(4):1225-1231

96 ChangJY, Komaki R, Lu C, et al. Phase 2 study of high-dose proton therapy with concurrent chemotherapy for unresectable stage III nonsmall cell lung cancer. Cancer 2011;117(20):4707-4713

97 Hoppe BS, Henderson R, Pham D, et al. Phase II trial of concurrent chemotherapy and proton therapy for stage 3 NSCLC. Int J Radiat Oncol Phys. 2016;95:517-519

98 Oshiro Y, Okumura T, Kurishima K, et al. High-dose concurrent chemo-proton therapy for Stage III NSCLC: preliminary results of a Phase II study. J Radiat Res (Tokyo) 2014;55(5):959-965

99 Vokes EE, Herndon JE II, Kelley MJ, et al; Cancer and Leukemia Group B. Induction chemotherapy followed by chemoradiotherapy compared with chemoradiotherapy alone for regionally advanced unresectable stage III Non-small-cell lung cancer: cancer and Leukemia Group B. J Clin Oncol 2007;25(13):1698-1704

100 Dionisi F, Croci S, Giacomelli I, et al. Clinical results of proton therapy reirradiation for recurrent nasopharyngeal carcinoma. Acta Oncol 2019;58(9):1238-1245

101 Ho JC, Nguyen QN, Li H, et al. Reirradiation of thoracic cancers with intensity modulated proton therapy. Pract Radiat Oncol 2018;8(1):58-65

102 Thorpe CS, Niska JR, Girardo ME, et al. Proton beam therapy reirradiation for breast cancer: Multi-institutional prospective PCG registry analysis. Breast J 2019;25(6):1160-1170

103 Boimel PJ, Berman AT, Li J, et al. Proton beam reirradiation for locally recurrent pancreatic adenocarcinoma. J Gastrointest Oncol 2017;8(4):665-674

104 Moningi S, Ludmir EB, Polamraju P, et al. Definitive hyperfractionated, accelerated proton reirradiation for patients with pelvic malignancies. Clin Transl Radiat Oncol 2019;19:59-65

105 Fernandes A, Berman AT, Mick R, et al. A prospective study of proton beam reirradiation for esophageal cancer. Int J Radiat Oncol Biol Phys 2016;95(1):483-487

106 Imber BS, Neal B, Casey DL, et al. Clinical outcomes of recurrent intracranial meningiomas treated with proton beam reirradiation. Int J Part Ther 2019;5(4):11-22 